\title{
Increase of Indicators of Vehicles' Smooth Running by Internal Suspension of Wheels
}

\author{
Vladimir Timofeev \\ Institute of Engineering Sciences \\ Pskov State University \\ Pskov, Russia \\ timofeev1097@yandex.ru \\ Evgeny Seleznev \\ Institute of Engineering Sciences \\ Pskov State University \\ Pskov, Russia \\ evgeniys84@yandex.ru
}

\author{
Alexander Enaev \\ Institute of Engineering Sciences \\ Pskov State University \\ Pskov, Russia \\ enaa53@mail.ru \\ Tatyana Klets \\ Institute of Humanities and Linguistic \\ Communications \\ Pskov State University \\ Pskov, Russia \\ kte63@yandex.ru
}

\author{
Anna Dmitrieva \\ Institute of Engineering Sciences \\ Pskov State University \\ Pskov, Russia \\ anna-listratova@rambler.ru
}

\begin{abstract}
The article discusses the relevance of increasing the smoothness of the movement of vehicles, and also substantiates the feasibility of using internal suspension of wheels in conjunction with a suspension and as an independent system.
\end{abstract}

Keywords - inelastic resistance coefficient, normal stiffness coefficient, the smoothness of vehicles' course, the wheel with internal springing.

\section{INTRODUCTION}

The operational properties of the vehicle characterize the performance of transport and special works. These properties determine the vehicle's adaptability to operating conditions, efficiency and ease of use. Improving performance is a priority in automotive design.

Smooth running is an important performance property. It largely affects the average speed, fuel consumption, ride comfort, safety of the transported cargo and the health of the driver and passengers from the impact of road irregularities. With an improvement in the smoothness of the course, an increase in the overhaul mileage of vehicles is observed.

When a car moves on an uneven road, not only vibrations of the body occur, but also vibrations of different frequencies caused by the interaction of the wheels with the supporting surface, the peculiarities of the operation of individual suspension elements, as well as aerodynamic forces. All this, in addition to the above- described consequences, leads to the occurrence of mechanical stresses in the suspension and the occurrence of noise both inside the car and outside, which in turn leads to noise pollution of the environment.

Various factors influence the ride smoothness: the type of suspension, the use of certain elastic and damping devices, the elasticity of pneumatic tires, and others.

Obviously, the problem of improving the ride quality indicators is urgent. In this regard, continuous improvement is carried out not only for individual parts of vehicles, but also for equipment for testing them in various conditions, innovative solutions and non-standard approaches to design are applied.

The vibration system of a car is a complex multi-mass system that includes sprung and unsprung masses. The first ones rely on the elastic element of the car suspension and perform low-frequency vibrations $(1 \ldots 1.5 \mathrm{~Hz})$, the second ones - have contact with the road surface and vibrate with high frequencies $\left(\begin{array}{lll}5.8 & \ldots & 12 \mathrm{~Hz}\end{array}\right)$. The car makes free vibrations when driving on a flat road after passing an obstacle. In fact, such vibrations are damped by the friction in the suspension and the resistance of the damping devices.

Suspension and pneumatic tires of the vehicle are responsible for reducing vibration load and elastic seats for drivers and passengers. 
The use of pneumatic tires improves the ride comfort due to their elasticity, smoothing and absorption capacity. Tires have a radial stiffness 6-12 times that of an elastic suspension element. They, along with the suspension, are involved in reducing the dynamic effects of road irregularities.

\section{APPLICATION OF WHEELS WITH INNER SPRINGS ON THE AUTOMOTIVE TRANSPORT}

An innovative solution was the creation of wheels with internal suspension, the elastic element of which is located between the wheel hub and the rim of the disc. Due to their compact size, the best indicators of absorption of the dynamic effects of road irregularities, the use of such structures is advisable on vehicles with a suspension installed or instead of a suspension. In the first case, there is an improvement in the smoothness of the movement, in the second, a simplification of the design and, as a result, an increase in reliability and a decrease in the cost of the product.

At Pskov State University, an experimental model of a wheel with internal suspension was created [1] Patent No. 2524269.

Studies of the smooth running of a vehicle equipped with such wheels have shown the effectiveness of their use. The wheel was installed on a car trailer instead of the standard suspension. At the same time, it was possible to maintain the values of the maximum vertical acceleration of the body without using the suspension. However, in the course of experiments, it turned out that the elastic element of the wheel is susceptible to lateral withdrawal. As a result of the action of the lateral force, the disc rim is displaced relative to the hub. As a result, the occurrence of vibrations in the horizontal plane is observed, which negatively affects the handling and safety of the vehicle (Fig. 1).

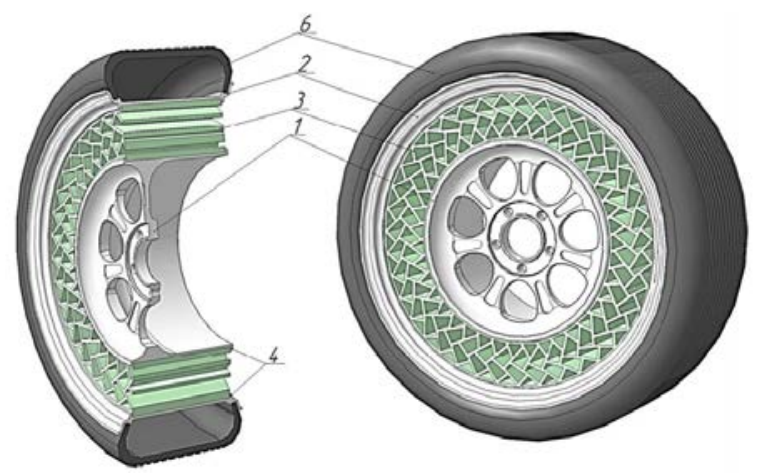

Fig. 1. Wheel with internal suspension. Patent No. 2524269

As a result of the analysis of the advantages and disadvantages of the wheel [1], a wheel with internal suspension was developed [2] Patent No. 2729851, the design of which is shown in Fig. 2. The wheel with internal suspension consists of separately made hubs with thrust plates 1 and a rim 2 connected by an elastic element 3 made of polymer material in the form of a closed ring of trapezoidal cross-section with end perforation. The rim of the disc, due to its trapezoidal shape, serves as an internal stop, partially preventing axial displacement of the hubs. The hubs with thrust plates are bolted together. Polymeric elastic element 3 is connected to the hub and rim by adhesion. The rim 2 has a pneumatic tire 4 .

The thrust plates serve to limit the axial movements of the hub 1 relative to the rim 2 and prevent oscillations in the horizontal plane. The thrust plates are installed at an angle that provides a portion of the horizontal force that causes the longitudinal displacement of the rim 2 relative to the hub, redistribution to the wheel axle, and thereby reducing the action of this force. This reduces the amount of longitudinal movement that will take place within the elastic deformation of the polymer material of the elastic element 3 located between the stop plates. The internal suspension parameters are calculated for a specific vehicle. Achieving the best smoothness of the ride is due to the coordination of the properties of the elastic element with the parameters of the vehicle. 


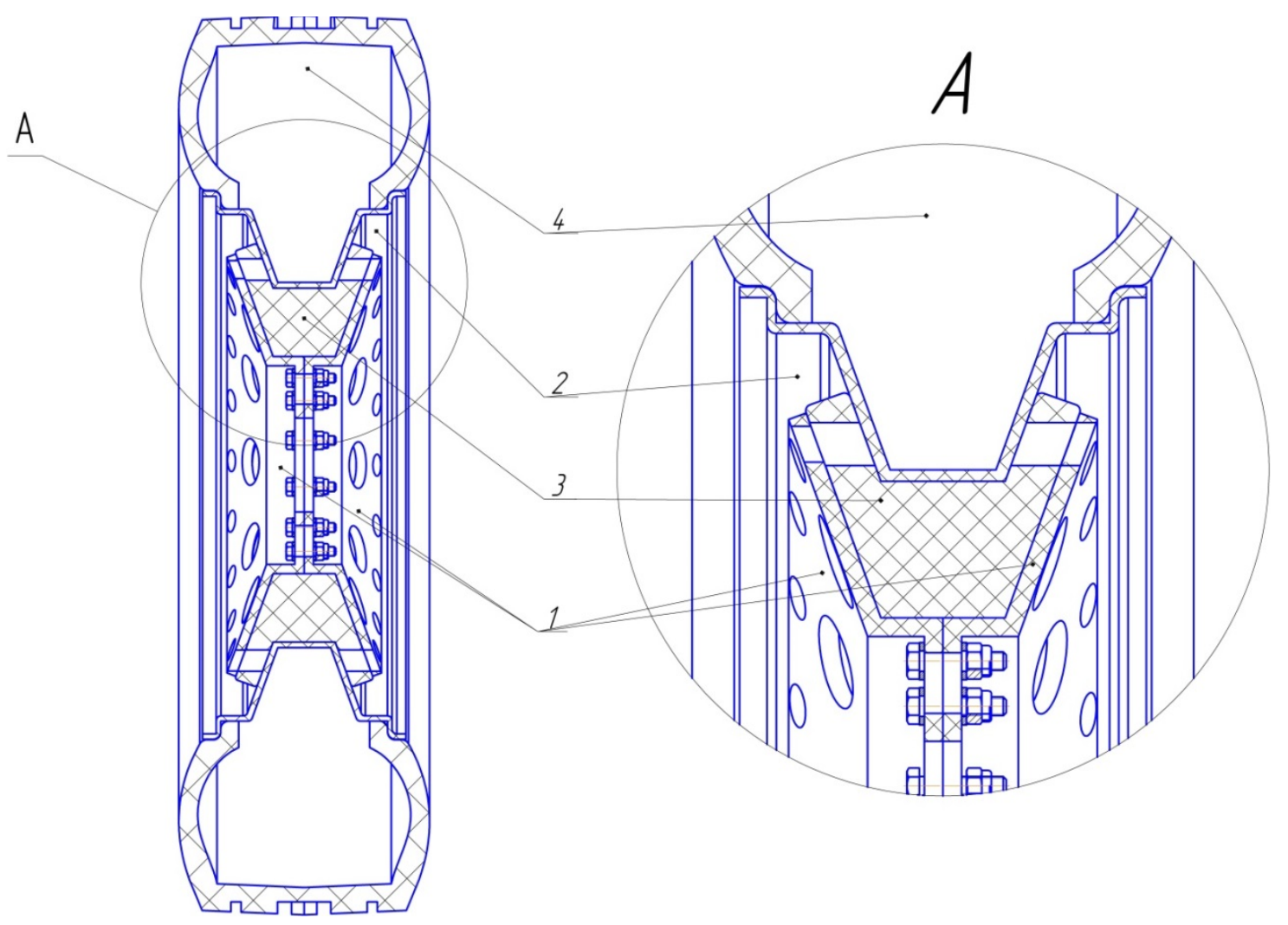

Fig. 2. Wheel with internal suspension. Patent No. 2729851.

With a decrease in the angle of inclination of the thrust plates, the normal load on the plates from the side of the action of centrifugal force decreases. The required amount of horizontal deformation of the elastic element is achieved. include:

Design requirements for an internally sprung wheel

- increasing the smoothness of the ride while maintaining controllability and stability of the vehicle by limiting the lateral movement of the wheel rim relative to the hub;

- reducing the load on the vehicle components due to the deformation of the elastic element.

The technical result is achieved by the fact that the elastic element is made of a polymeric material in the form of a closed ring with end perforation and is partially located between the thrust plates of the wheel hub and the rim of the disk. The thrust plates are angled to reduce the horizontal force. The amount of possible movement of the rim relative to the hub is determined by the end deformation of the polymer material located between the thrust plates. Improvement of the smoothness indicators is provided due to the possibility of deformation of the polymer elastic element.

Due to its trapezoidal shape, the rim of the disc acts as an internal thrust bearing. It should be borne in mind that the wheel is a complex elastic system consisting of several elements. The thrust plates are parallel and spaced from the inner rim bearing surface. The distance is determined by the angle of inclination of the thrust plates. The angle of inclination of the plates to the horizon ranges from $30^{\circ}$ to $90^{\circ}$.

The elastic and damping properties of the wheel can vary depending on the end perforation and the geometry of the elastic element. It is also proposed to adjust the stiffness of the wheel by using materials with different properties. So, for the production of an elastic element, it is possible to select urethane elastomers of different hardness. This makes it possible to match the internal suspension properties and vehicle-specific characteristics to achieve the best ride quality.

\section{THEORETICAL MODEL OF VIBRATIONS OF A VEHICLE EQUIPPED WITH INNER SPRING WHEELS}

A diagram of an oscillating system equivalent to a suspension with a wheel containing internal suspension is shown in Fig. 3. 


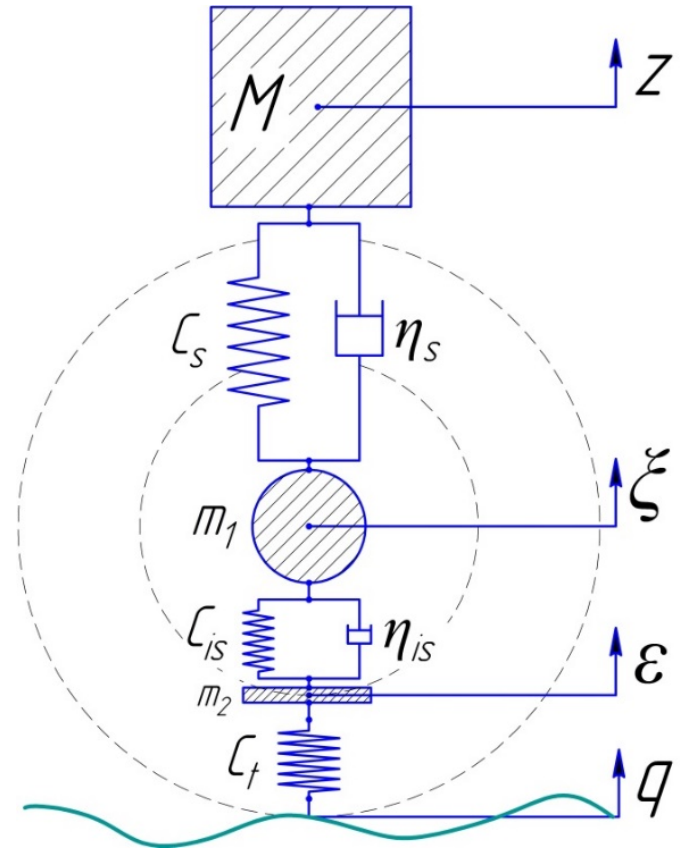

Fig. 3. Oscillation system equivalent to a suspension with an internally cushioned wheel.

When drawing up this scheme, the images and designations generally accepted in the theory of ride and cushioning are preserved:

$\mathrm{M}$ - sprung mass, kg

$m_{1}$ - the first unsprung mass, $\mathrm{kg}$

$m_{2}$ - the second unsprung mass, $\mathrm{kg}$

$\mathrm{z}$ - vertical movement of the sprung mass, $\mathrm{kg}$

$\xi$ - vertical movement of the first unsprung mass, $m$

$\varepsilon$ - vertical movement of the second unsprung mass, $\mathrm{m}$ $\mathrm{N} / \mathrm{m}$

$C_{S^{-}}$normal stiffness of the elastic suspension element,

$C_{i s}$ - normal stiffness of the elastic element of the inner suspension, $\mathrm{N} / \mathrm{m}$

$C_{t}$ - normal tire stiffness, $\mathrm{N} / \mathrm{m}$

$\eta_{s}$ - suspension inelastic resistance coefficient, $\mathrm{N}^{*} \mathrm{~s} / \mathrm{m}$

$\eta_{i s}$ - coefficient of inelastic resistance of internal suspension, $\mathrm{N}^{*} \mathrm{~s} / \mathrm{m}$

In this case:

$M \ddot{z}$ - sprung mass inertia, $\mathrm{N}$

$c_{S}(z-\xi)-$ suspension elastic force, $\mathrm{N}$

$\eta_{s}(\dot{z}-\dot{\xi})-$ inelastic suspension force, $\mathrm{N}$

$m_{1} \ddot{\xi}$ - inertia force of the first unsprung mass, $\mathrm{N}$

$c_{i s}(\xi-\varepsilon)-$ internal suspension force, $\mathrm{N}$ $\eta_{i s}(\dot{\xi}-\dot{\varepsilon})-$ internal suspension inelastic resistance force, $\mathrm{N}$

$m_{2} \ddot{\varepsilon}$ - inertia force of the second unsprung mass, $\mathrm{N}$

$c_{t}(\varepsilon-q)-$ tire elastic force, $\mathrm{N}$

Thus, the oscillations of the system can be represented as:

$$
\left\{\begin{array}{c}
M \ddot{z}+c_{s}(z-\xi)+\eta_{s}(\dot{z}-\dot{\xi})=0 ; \\
m_{1} \ddot{\xi}+c_{i s}(\xi-\varepsilon)+\eta_{i s}(\dot{\xi}-\dot{\varepsilon})- \\
-c_{s}(z-\xi)-\eta_{s}(\dot{z}-\dot{\xi})=0 ; \\
m_{2} \ddot{\varepsilon}+c_{t}(\varepsilon-q)-c_{i s}(\xi-\varepsilon)- \\
\quad-\eta_{i s}(\dot{\xi}-\dot{\varepsilon})=0 .
\end{array}\right.
$$

Dividing the left and right sides of the equations of system (1) by $M, m_{1}$ and $m_{2}$ respectively we get:

$$
\left\{\begin{array}{c}
\ddot{z}+\frac{c_{s}}{M}(z-\xi)+\frac{\eta_{s}}{M}(\dot{z}-\dot{\xi})=0 ; \\
\ddot{\xi}+\frac{c_{i s}}{m_{1}}(\xi-\varepsilon)+\frac{\eta_{i s}}{m_{1}}(\dot{\xi}-\dot{\varepsilon})- \\
-\frac{c_{s}}{m_{1}}(z-\xi)-\frac{\eta_{s}}{m_{1}}(\dot{z}-\dot{\xi})=0 ; \\
\ddot{\varepsilon}+\frac{c_{t}}{m_{2}}(\varepsilon-q)-\frac{c_{i s}}{m_{2}}(\xi-\varepsilon)- \\
-\frac{\eta_{i s}}{m_{2}}(\dot{\xi}-\dot{\varepsilon})=0 .
\end{array}\right.
$$

Taking into account the well-known designations

$$
\begin{aligned}
\frac{c_{s}}{M} & =\Omega^{2} ; \\
\frac{c_{i s}}{m_{1}} & =\omega_{1}^{2} ; \\
\frac{c_{i s}}{m_{2}} & =\omega_{2}^{2} ; \\
\frac{\eta_{s}}{M} & =2 k ; \\
\frac{\eta_{i s}}{m_{1}} & =2 k_{1} .
\end{aligned}
$$

Introducing the notation

$$
\begin{gathered}
\frac{m_{1}}{M}=\mu_{1} ; \\
\frac{m_{1}}{M}=\mu_{2} .
\end{gathered}
$$

we get:

$$
\begin{aligned}
& \frac{c_{s}}{m_{1}}=\frac{c_{s}}{\mu_{1} M}=\frac{\Omega^{2}}{\mu_{1}} ; \\
& \frac{\eta_{s}}{m_{1}}=\frac{\eta_{s}}{\mu_{1} M}=\frac{2 k}{\mu_{1}} ; \\
& \frac{c_{i s}}{m_{2}}=\frac{c_{i s}}{\mu_{2} m_{1}}=\frac{\omega_{1}^{2}}{\mu_{2}} ; \\
& \frac{\eta_{i s}}{m_{2}}=\frac{\eta_{i s}}{\mu_{2} m_{1}}=\frac{2 k_{1}}{\mu_{2}} .
\end{aligned}
$$

Then equations (2), taking into account the adopted designations, take the form: 
Environment. Technology. Resources. Rezekne, Latvia Proceedingsofthe $13^{\text {th }}$ InternationalScientificandPractical Conference. Volume 3, 343-347

$$
\left\{\begin{array}{c}
\ddot{z}+\Omega^{2}(z-\xi)+2 k(\dot{z}-\dot{\xi})=0 ; \\
\ddot{\xi}+\omega_{1}^{2}(\xi-\varepsilon)+2 k_{1}(\dot{\xi}-\dot{\varepsilon})- \\
-\frac{\Omega^{2}}{\mu_{1}}(z-\xi)-\frac{2 k_{1}}{\mu_{2}}(\dot{z}-\dot{\xi})=0 ; \\
\ddot{\varepsilon}+\omega_{2}^{2}(\varepsilon-q)-\frac{\omega_{1}^{2}}{\mu_{2}}(\xi-\varepsilon)- \\
-\frac{2 k_{1}}{\mu_{2}}(\dot{\xi}-\dot{\varepsilon})=0 .
\end{array}\right.
$$

or

$$
\left\{\begin{array}{c}
\ddot{z}+2 k \dot{z}+\Omega^{2} z-2 k \dot{\xi}-\Omega^{2} \xi=0 ; \\
\ddot{\xi}+2\left(k_{1}+\frac{k}{\mu_{1}}\right) \dot{\xi}+\left(\omega_{1}^{2}+\frac{\Omega^{2}}{\mu_{1}}\right) \xi- \\
-2 k_{1} \dot{\varepsilon}-\omega_{1}^{2} \varepsilon-\frac{2 k}{\mu_{1}} \dot{z}-\frac{\Omega^{2}}{\mu_{1}} z=0 ; \\
\ddot{\varepsilon}+\frac{2 k_{1}}{\mu_{2}} \dot{\varepsilon}+\left(\omega_{2}^{2}+\frac{\omega_{1}^{2}}{\mu_{2}}\right) \varepsilon-\frac{2 k_{1}}{\mu_{2}} \dot{\xi}- \\
-\frac{\omega_{1}^{2}}{\mu_{2}} \xi=\omega_{2}^{2} q .
\end{array}\right.
$$

The proposed mathematical model (4) will allow at the design stage to determine, by the minimum value of the vertical displacements of the sprung masses, the optimal parameters characterizing the elastic and damping properties of the internal suspension.

\section{CONCLUSIONS}

Thus, due to the use of the described product, it is assumed that the material consumption of the production of vehicles will be reduced, as a result of the exclusion of the suspension from the design. When using wheels with internal suspension in conjunction with the suspension, it is assumed that the dynamic effects on the vehicle parts and the road surface will be reduced. This will have a positive effect on the service life of both the vehicle and the road network.

\section{REFERENCES}

[1] A. A. Enaev and S. B. Manfanovsky, "Wheel with internal suspension No. 20121262213/11," Patent No. 2,524,269, Russian Federation, IPC B60B 9/26. Declared June 22, 2012; Publ. Dec. 27, 2013, Bul. No. 21.

[2] A. A. Enaev, V. S. Timofeev, and P. N. Maltsev, "Wheel with internal suspension. No. 2019133497", Patent No. 2729851 Russian Federation, IPC B60B 9/26. Declared October 21, 2019, Publ. August 12, 2020, Bul. No. 23.

[3] A. A. Enaev, "Fundamentals of the theory of vehicle vibrations during braking and its applications." M.: Mashinostroenie, 2002, 341 p.

[4] S. B. Manfanovsky, "Improving the smoothness of the movement of transport and transport-technological machines by internal suspension of wheels," PhD thesis, Pskov, 2019. 